\title{
Aktuelle Informationen aus dem Ressort
}

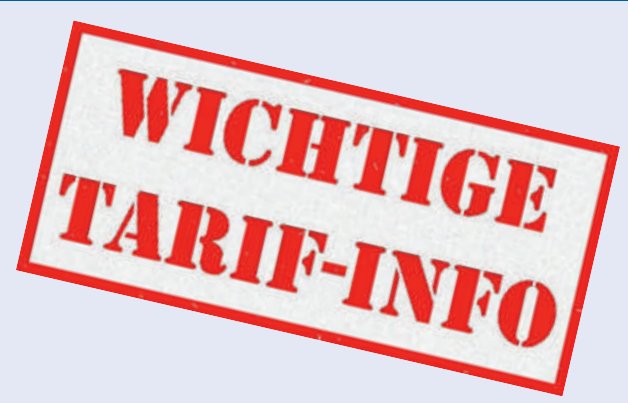

\author{
Susanne Christen ${ }^{\text {, }}$ \\ Irène Marty ${ }^{b}$, \\ Thomas Kesslerc, \\ Kerstin Rutsch \\ a Dr. med., Mitarbeiterin \\ Ressort Ambulante Tarife und \\ Verträge Schweiz \\ b Leiterin Ressort Ambulante \\ Tarife und Verträge Schweiz \\ c Mitarbeiter/in Ressort \\ Ambulante Tarife und \\ Verträge Schweiz
}

Korrespondenz:

FMH Ressort Ambulante Tarife und Verträge Schweiz

Froburgstrasse 15

CH-4600 Olten

Tel. 0313591230

Fax 0313591238

tarife.ambulant[at]fmh.ch

\section{Delegierte Psychotherapie - Änderung der Kriterien für die Spartenanerkennung}

Die Kapitelinterpretation der Delegierten Psychotherapie in der Arztpraxis (Kapitel 02.03 TARMED) und damit auch ein Kriterium für diese Spartenanerkennung wurde leicht modifiziert. So gilt ab 1. April 2013: «Die delegierte Psychotherapie wird pro Arzt auf maximal 100 Wochenstunden beschränkt. Die Aufsichtspflicht des delegierenden Arztes muss gewährleistet sein.»

Die etwas schwer verständliche, frühere Regelung von «... 4 Therapeuten und/oder maximal 100 Wochenstunden ...» ist damit nun klar definiert. Diese Beschränkung betrifft nur den Zeitaspekt (max. 100 Stunden pro Woche), unabhängig von der Anzahl der angestellten Therapeuten. Die neue Regelung ist mit dem PIK-Entscheid I-13001 Anpassung KI-02.03-1 (Verrechenbarkeit delegierte Psychotherapie) rechtskräftig.

Die Liste aller publizierten Entscheide der Paritätischen Interpretationskommission (PIK) sind unter dem folgenden Link abrufbar: www.tarmedsuisse.ch $\rightarrow$ PIK-Interpretationen $\rightarrow$ Version 1.54 vom 1.4.2013.

\section{Abbildung 1}

Im TARMED-Browser (www.tarmedsuisse.ch/86.html) ist pro Leistung ersichtlich, zu welcher Sparte sie zugeordnet ist. (Quelle: TARMED-Browser)

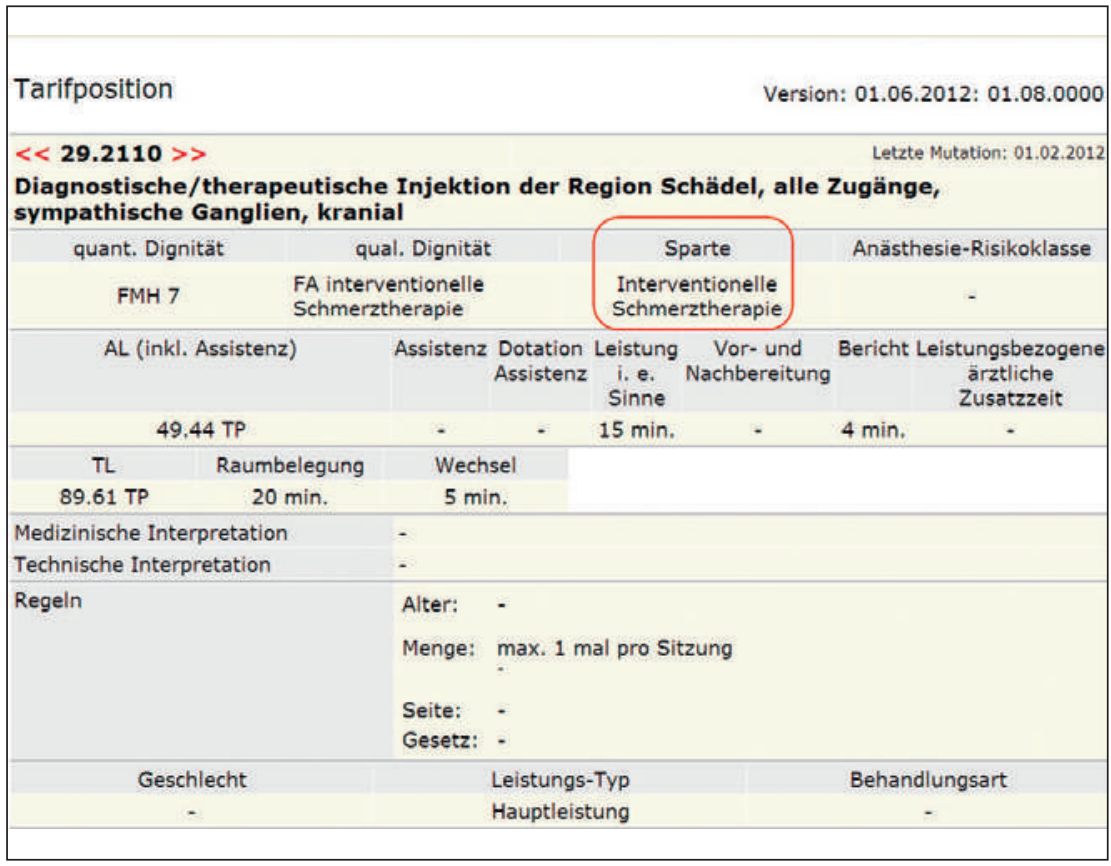

Spartenanerkennung Interventionelle Schmerztherapie ab 1.6.2013 in Kraft

Die neue Spartenanerkennung Interventionelle Schmerztherapie, die mit der TARMED Version 1.08 zusammen mit den revidierten Leistungen der Schmerztherapie (Kapitel 29) eingeführt wurde, ist nun ab 1. Juni 2013 gültig und kann von den Versicherungen überprüft werden.

Neu wird für Positionen aus dem Unterkapitel 29.06 «Interventionelle Schmerzdiagnostik und -therapie» eine sogenannte Spartenanerkennung als Voraussetzung für die Abrechnung verlangt.

Weitere Informationen dazu und die notwendigen Formulare finden Sie auf unserer Homepage unter www.fmh.ch $\rightarrow$ Ambulante Tarife $\rightarrow$ TARMED Sparten - Interventionelle Schmerztherapie.

\section{Spartenanerkennung - Was ist das?}

Verschiedene Leistungen im Tarifwerk TARMED verlangen für ihre Abrechnung eine sogenannte Spartenanerkennung. Die für den Praxisarzt wichtigsten Spartenanerkennungen sind:

- Ambulante Chirurgie (OP I und Praxis-OP)

- Delegierte Psychotherapie

- Interventionelle Schmerztherapie

- Betriebsstelle Radiologie (nur für selbständige Institute)

Wenn Sie TARMED-Leistungen in diesen Bereichen (vgl. Sparte bei einer Leistung im TARMED-Browser) durchführen und zulasten der Sozialversicherungen abrechnen wollen, müssen Sie eine Spartenanerkennung beantragen. Dazu müssen Sie den entsprechenden Selbstdeklarationsbogen ausfüllen und an die FMH, Ressort Ambulante Tarife und Verträge Schweiz, in Olten senden.

Die Grundlage der Spartenanerkennung findet sich im Spartenkonzept, das von der Paritätischen Kommission Dignität und Spartenanerkennung (PaKoDig) erstellt und nach Bedarf aktualisiert wird.

Weitere Informationen sowie alle Formulare zur Beantragung einer Spartenanerkennung finden Sie auf unserer Homepage unter www.fmh.ch $\rightarrow$ Ambulante Tarife $\rightarrow$ TARMED Sparten.

\section{Delegations- und/oder Anordnungsmodell für Leistungen der nichtärztlichen Psychotherapeut(inn)en}

Im BAG laufen die inhaltlichen Vorarbeiten für eine 
Änderung der Vergütung der nichtärztlichen Psychotherapie bei den Psychotherapeutinnen und -therapeuten als selbständige Leistungserbringer durch die obligatorische Krankenpflegeversicherung. Ein erstes Hearing mit allen Betroffenen fand am 9. April 2013 statt. In den kommenden Wochen wird diskutiert, wie genau die inhaltliche Ausgestaltung einer solchen neuen Regelung aussehen soll.

\section{Heutige Regelung: Delegation}

Gemäss der ständigen Rechtsprechung des ehemaligen Eidgenössischen Versicherungsgerichts seit Mai 1981 ist die delegierte Psychotherapie eine Pflichtleistung der Krankenversicherer, wenn die Psychotherapeutinnen und Psychotherapeuten in den Praxisräumen des Arztes oder der Ärztin, unter seiner/ ihrer direkten Aufsicht und Verantwortung und im Rahmen eines Anstellungsverhältnisses arbeiten. Die Leistung muss überdies überhaupt delegierbar sein. Solche Leistungen gelten rechtlich als vom Arzt erbracht und dürfen bzw. müssen von ihm im eigenen Namen zuhanden der OKP verrechnet werden.

Diese oben genannten Kriterien liegen auch der sogenannten Spartenanerkennung Delegierte Psychotherapie im TARMED zugrunde, wobei sich der Arzt über seine Kompetenzen ausweisen muss, delegierte Leistungen abrechnen zu können (mit dem Facharzttitel Psychiatrie und Psychotherapie, Kinder- und Jugendpsychiatrie und -psychotherapie, oder dem Fähigkeitsausweis Delegierte Psychotherapie FMPP).

\section{Geplante Regelung: Anordnung}

Vorgesehen ist, dass die nichtärztlichen Psychotherapeut(inn)en (gemäss Psychologieberufsgesetz PsyG) als neue, auf ärztliche Anordnung hin selbständig tätige Leistungserbringer in der Verordnung über die Krankenversicherung (KVV) aufgenommen werden. In der KVV müssen demzufolge die Zulassungsvoraussetzungen dieser Leistungserbringer formuliert und in der Krankenpflege-Leistungsversordnung (KLV) müssen die von der obligatorischen Krankenpflegeversicherung (OKP) zu übernehmenden Leistungen umschrieben werden.

Die FMH vertritt die Meinung, wenn nichtärztliche Psychotherapeuten eigenverantwortlich in eigener Praxis arbeiten dürfen, sollen sie auch frei in der Entscheidung sein, sich in einer HMO, in einer Quartiergruppenpraxis usw. anstellen zu lassen. Wenn nötig müssen die gesetzlichen Grundlagen ge- schaffen werden, dass Anstellungsverhältnisse auch in Zukunft möglich sind.

\section{Krankenpflege-Leistungsverordnung KLV Anhang 1 - kein abschliessender Leistungs- katalog!}

Gemäss Beschluss des Bundesgerichtes aus dem Jahr 2003 (BGE 129 V 167), ist Anhang 1 der KLV weder eine Positiv- noch eine Negativliste. Diese enthält lediglich die von der Eidgenössischen Kommission für allgemeine Leistungen und Grundsatzfragen (ELGK) geprüften Leistungen, die von der obligatorischen Krankenpflegeversicherung im Behandlungsfall zu übernehmen, nicht zu übernehmen, oder nur unter bestimmten Voraussetzungen zu übernehmen sind.

Bei Behandlungen, die bisher nicht Gegenstand einer Prüfung durch die ELGK waren, greift die gesetzliche Vermutung Platz, dass die ärztlich erbrachte Leistung den gesetzlichen Prinzipien der wissenschaftlich nachgewiesenen Wirksamkeit, Wirtschaftlichkeit und Zweckmässigkeit (Art. 32 Abs. $1 \mathrm{KVG}$ ) entspricht. Ergo sind Behandlungen, die nicht im Anhang 1 der KLV erwähnt sind, bei Krankheit von der obligatorischen Krankenpflegeversicherung zu übernehmen.

\section{Jetzt vormerken: Tarifdelegierten-Tag} Mittwoch, 23. Oktober 2013, Hotel Bern Die Veranstaltung richtet sich an die Tarifdelegierten der medizinischen Fachgesellschaften und kantonalen Ärztegesellschaften.

Das Ressort FMH Ambulante Tarife und Verträge Schweiz informiert an diesem Anlass zu Themen aus den unterschiedlichen Arbeitsbereichen, wie zum Beispiel über den Stand der Tarifrevision, zum Praxislabor und generell zur aktuellen Situation im Tarifbereich.

\section{Neuer Webauftritt}

Das Ressort Ambulante Tarife und Verträge Schweiz der FMH hat seinen Webauftritt überarbeitet. Die neue Rubrik «Ambulante Tarife» finden Sie unter www.fmh.ch. Hier haben wir wichtige Informationen kurz und bündig für Sie zusammengestellt, Detailinformationen stehen für den interessierten Leser mit einem Klick «Mehr» zur Verfügung. 